Review article

\title{
Effect of multiple extrusions and influence of PP contamination on the thermal characteristics of bottle grade recycled PET
}

\author{
Bachir Itim, Mathew Philip* \\ Sir John Cass Faculty of Art, Architecture and Design, London Metropolitan University, London E1 1LA, UK
}

\section{A R T I C L E I N F O}

\section{Article history:}

Received 4 January 2015

Received in revised form

15 March 2015

Accepted 5 April 2015

Available online 11 April 2015

\section{Keywords:}

Bottle grade recycled PET

PP contamination

Chain scission

Cross-linking

Crystallinity

\begin{abstract}
A B S T R A C T
The thermal characteristics of bottle grade recycled PET pellets contaminated with $5 \%$ PP prepared by multiple extrusions were studied by differential scanning calorimetry (DSC) at constant heating rate of $10{ }^{\circ} \mathrm{C} / \mathrm{min}$ for various extrusion cycles. The peak temperature of crystallization $\boldsymbol{T}_{\boldsymbol{c}}$, the enthalpy of crystallization $\boldsymbol{\Delta} \boldsymbol{H}_{\boldsymbol{c}}$ and the degree of crystallinity $\boldsymbol{X}_{\boldsymbol{c}}$ were found to be dependent on the number of extrusion cycles. From the dependence on extrusion cycles, the analysis of the crystallization mechanism by Avrami model has been determined, the Avrami exponent $\boldsymbol{n}$ and the rate constant $\boldsymbol{Z}_{\boldsymbol{t}}$ were calculated and the influence of the 5\% PP on the bottle grade recycled PET matrix during multiple extrusions and its effect on crystallinity was thoroughly discussed.
\end{abstract}

() 2015 Elsevier Ltd. All rights reserved.

\section{Introduction}

Bottle grade recycled PET referred to in future as rPET-bg can replace and be used as an alternative to virgin PET and reducing environmental issues related to landfilling and incineration. rPETbg is considered to lose its inherited properties through recycling and reprocessing cycles. During recycling, rPET-bg gets cross contaminated with other polymeric materials, especially PP. This cross contamination is a major issue in rPET-bg recycling industry because of its influence on the characteristics of the final produce. Furthermore, during reprocessing of the contaminated rPET-bg, the transparency of the final produce is altered as a result of contamination. The present study, focussed on the influence of PP contamination on the thermal characteristics of rPET-bg, especially crystallinity which dictates transparency in PET, and its importance in bottle manufacturing. Crystallization kinetics of engineering grades have been extensively studied $[1,2]$. However, studies on recycled materials such as rPET-bg have been rare. The influence of polymeric contaminants, such as PP, on the crystallization of rPET is particularly important as PP bottle tops are easily mixed with rPETbg when recycling. Their presence in small amounts in rPET-bg can significantly affect crystallinity during multiple extrusions. Some

\footnotetext{
* Corresponding author. Tel.: +44 2073202843.

E-mail address: M.Philip@londonmet.ac.uk (M. Philip).
}

researchers have studied the degradation of rPET during processing and concluded that chain scission can occur and that formation of grafted copolymers and crystallization can be facilitated [4,5] and others concluded that during repetitive extrusion, chain scission predominated and no chain branching or cross-linking were observed which decreased capabilities and crystallinity [7,8], whereas others concluded that cross-linking and chain branching occur during extrusion $[9,10]$. This research study investigates the influence of thermo-mechanical degradation during repetitive extrusion cycles on the crystallinity of non-contaminated and contaminated rPET matrix with 5\% PP. Furthermore, the Avrami model may properly describe the behaviour of crystallization of the non-contaminated and contaminated rPET under isothermal crystallization process.

\section{Materials and method}

The rPET-bg flakes, mixture of multicolored flakes were supplied by Closed Loop Recycling Ltd [11].

The PP addition selected was the grade 7011L1 manufactured by ExxonMobil Chemical with properties given in Table 1. Samples of non contaminated rPET-bg and others with 5\% PP were prepared by multiple extrusions for 5 cycles using a Leistritz twin screw extrusion machine. The samples were dried for $5 \mathrm{~h}$ at a temperature of $120{ }^{\circ} \mathrm{C}$ and all processed under same temperature range of $250-270^{\circ} \mathrm{C}$ and a screw speed of $120 \mathrm{rpm}$. 
Table 1

Some properties of rPET $^{\mathrm{d}}$ and PP grade 7011L1.

\begin{tabular}{lll}
\hline Property & rPET & PP \\
\hline $\mathrm{T}_{\mathrm{m}}{ }^{\mathrm{a}}$ (DSC) & $254{ }^{\circ} \mathrm{C}$ & $161{ }^{\circ} \mathrm{C}$ \\
$\mathrm{T}_{\mathrm{c}}{ }^{\mathrm{b}}(\mathrm{DSC})$ & $207{ }^{\circ} \mathrm{C}$ & $11{ }^{\circ} \mathrm{C}$ \\
$\mathrm{MFR} / \mathrm{I}^{\mathrm{C}}\left(230^{\circ} \mathrm{C} / 2.16 \mathrm{~kg}\right)$ & $61 \mathrm{~g} / 10 \mathrm{~min}$ & $1 \mathrm{~g} / 10 \mathrm{~min}$ \\
Tensile strength & $19 \mathrm{MPa}$ & $26 \mathrm{MPa}$ \\
\hline
\end{tabular}

${ }^{\text {a }}$ Melting temperature.

b Peak crystallization temperature.

c Melt flow rate/index.

d Own test results of rPET flakes.

e ExxonMobil data.

The thermal characteristics of the component materials and the influence of PP on the crystallization behavior of rPET-bg were evaluated using a TA Instrument differential scanning calorimeter, DSC2010 and software V4.4E and Universal V3.9A. The calorimeter was calibrated using indium. The average sample weight was approximately $10 \mathrm{mg}$ and 3 DSC runs of each material were performed. The samples were heated and cooled at a constant rate of $10{ }^{\circ} \mathrm{C} /$ min respectively over temperature ranges of $50-300{ }^{\circ} \mathrm{C}$ and $300-50{ }^{\circ} \mathrm{C}$ and isothermally kept above $300{ }^{\circ} \mathrm{C}$ for $2 \mathrm{~min}$ to complete melting.

The Avrami Equation [1-12] describing the development of crystallinity as function of time, which was used to investigate the dependence of crystallinity on the number of extrusion cycles in this study, is shown below:

$1-X_{t}=\exp \left(-Z_{t} t^{n}\right)$

Where: $\boldsymbol{X}_{\boldsymbol{t}}$ is the relative crystallinity at time $\boldsymbol{t}, \boldsymbol{n}$ and $\boldsymbol{Z}_{\boldsymbol{t}}$ are respectively the Avrami exponent and the rate constant and both of them are diagnostic of the crystallization mechanism.

Equation (1) can be rewritten as follow:

$\ln \left[-\ln \left(1-X_{t}\right)\right]=n \ln t+\ln Z_{t}$

Graphically, a double log plot of $\left(\ln \left[-\ln \left(1-X_{t}\right)\right]\right.$ Vs $\left.\ln t\right)$ generates 2 linear sections relative to primary and secondary processes. The Avrami equation applies only for the primary process. From the equation of the linear portion of the primary process, $\boldsymbol{n}$ is the slope and the value of $\boldsymbol{Z}_{\boldsymbol{t}}$ is calculated from the intercept, $\ln \boldsymbol{Z}_{\boldsymbol{t}}$.

\section{Results and discussion}

The thermal analysis at a cooling rate of $10{ }^{\circ} \mathrm{C} / \mathrm{min}$ of the extruded samples at various cycles $(1-5)$ showed that the crystallization temperatures of non contaminated rPET-bg and PP contaminated rPET-bg fluctuated respectively between 204.5 and $205.3^{\circ} \mathrm{C}$ and between 205.6 and $207.2^{\circ} \mathrm{C}$ as shown in Fig. 1, which indicates that the crystallization temperature decreased slightly on second extrusion after which it remained constant. The presence of $5 \%$ PP does increase the crystallization temperature by approximately $2{ }^{\circ} \mathrm{C}$, indicating melt thermo-mechanical degradation but also probable reactions between relevant macro-radicals in generating grafted copolymers [6].

The enthalpy of crystallization of non contaminated rPET-bg fluctuated with a tendency of decreasing from 43.4 to $35.2 \mathrm{~J} \mathrm{~g}^{-1}$, whereas that of PP contaminated rPET-bg decreased orderly from 34.7 to $7.7 \mathrm{~J} \mathrm{~g}^{-1}$ as shown in Fig. 2. This showed clearly the slow crystallization process in the PP contaminated rPET-bg compared with non contaminated rPET-bg meaning that the presence of PP even at small amount induced reduction of exothermic heat due to a reduction in crystallinity following multiple extrusions.

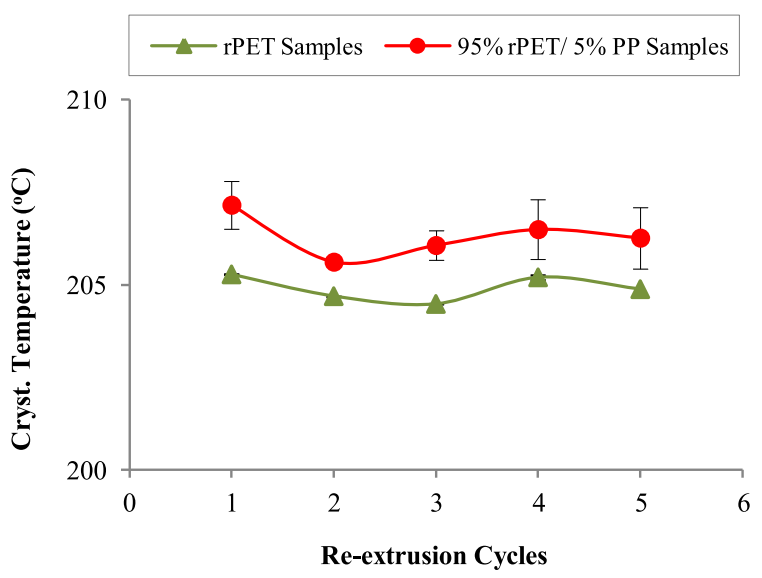

Fig. 1. Curves of crystallization temperatures Vs re-extrusion cycles.

Also, the \% crystallinity of non contaminated rPET-bg fluctuated randomly between 31.0 and $25.5 \%$ but in the PP contaminated rPETbg decreased orderly from 24.8 to $5.5 \%$ as shown in Fig. 3. One can see the proportional relationship of enthalpy of crystallization and $\%$ crystallinity.

Reducing crystallinity in PET bottle manufacturing is of paramount importance in enhancing bottle clarity or transparency. In real time bottle processing, during the cooling stage, the nucleation and growth processes of PET spherulites are the main factors governing the degree of crystallinity in the final product. The process of crystallization is initiated by the formation of primary nuclei, which is considered the starting point; nuclei form within small regions of the entangled and randomly distributed polymer chains to produce folded layers of ordered chains [13]. Then, crystallization propagates to form primary spherulites or crystallites. The formation of spherulites continues until the peak crystallization temperature, $\mathbf{T}_{\mathbf{c}}$, is reached. Then, secondary nucleation and secondary crystallization take place up to the end of the process. For the primary nuclei to form, the free energy barrier associated with the polymer chains disentangling and straightening must be crossed; the larger the fold period, the greater the barrier and the slower the rate of nucleation. In most cases, crystallinity starts on the surfaces of impurities or on deliberately added nucleating agents. Because no nucleating agents were added to this contaminated rPET-bg, the 5\% PP contributed in reducing the interfacial free energy necessary for crystallization and reduced the ease of disentanglement and straightening of the chains.

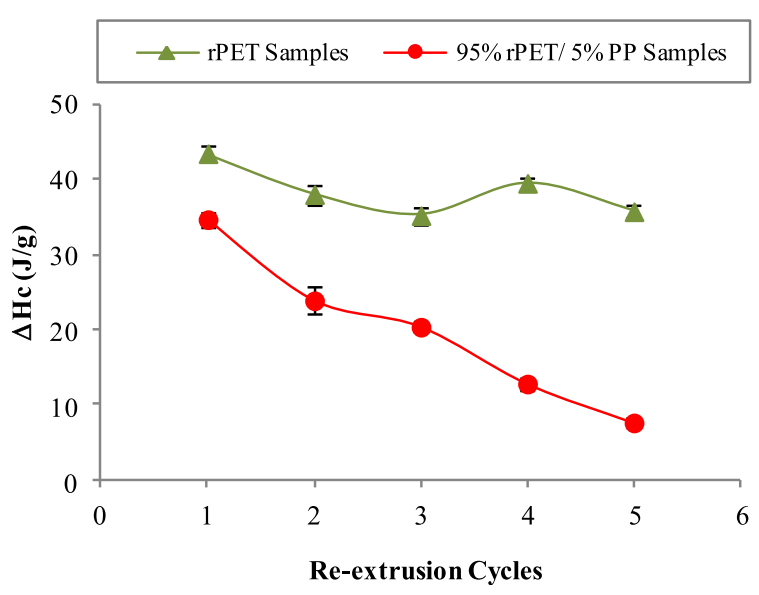

Fig. 2. Curves of heat of crystallizations at various re-extrusion cycles. 


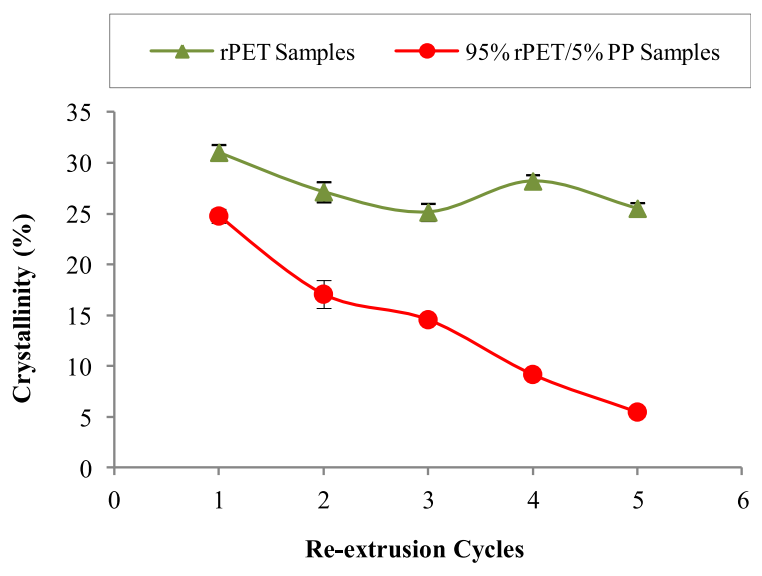

Fig. 3. Curves of crystallinity at various re-extrusion cycles.

As a result of this investigation, it was shown that the presence of 5\% PP contributed in lowering the degree of crystallinity in the rPET-bg matrix when subjected to multiple extrusions.

During multiple extrusions, both non contaminated and contaminated rPET-bg showed reduction in screw speed with increased extrusion cycles. This indicated that chain scission also influenced the materials flow characteristics and highly ordered chain orientation led to ease of processability in both cases. In comparison, the gap in screw speed decrease was larger with the PP contaminated rPET-bg than with non contaminated one as shown in Fig. 4, showing that chain scission and chain orientation, achieved in the convergent flow at the end of the die, were happening simultaneously, the latter was promoted by the flow property of PP which helped reducing the melt viscosity.

Furthermore, the screw \% torque shown in Fig. 5 complied with the processing rule of the inverse proportionality of screw speed and \% torque and it is clear to see that the gap in \% torque increase is larger with the PP contaminated rPET-bg than with the non contaminated one indicating that the PP contaminant generated certain degree of cross-linking resulting in an increase of viscosity despite the continuous chain scission due to thermo-mechanical degradation during multiple extrusions; this was in line with what was reported respectively [4-6] that during processing not only chain scission but also chain extension, grafted copolymers and crosslinking can occur and contradicted $[7,8]$ that only chain scission predominated and no chain branching or cross-linking were observed during multiple extrusions.

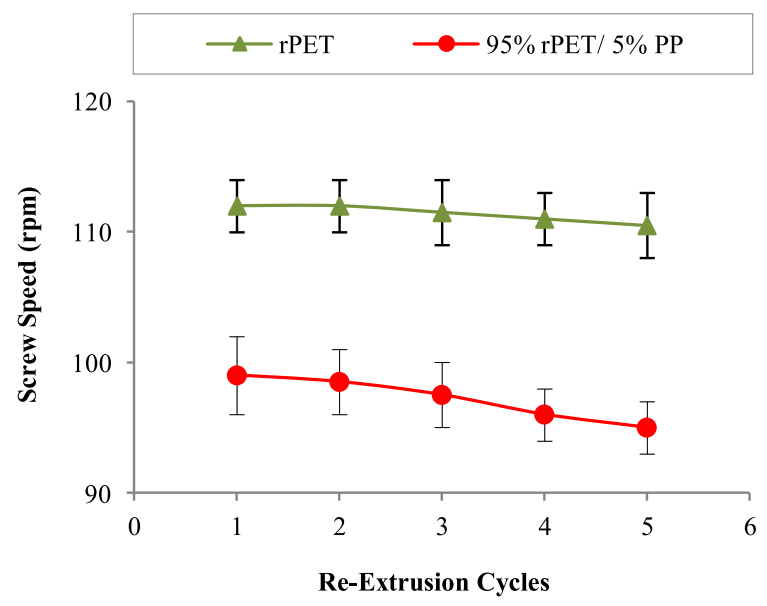

Fig. 4. Curves of screw speeds Vs re-extrusion cycles.

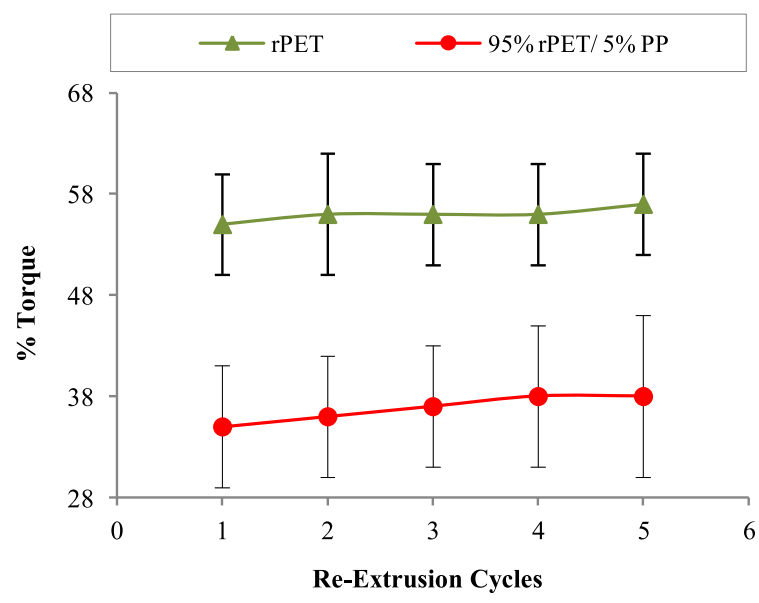

Fig. 5. Curves of \% torques Vs re-extrusion cycles.

Clearly, there is indication of competition between chain scission and cross-linking in the contaminated rPET-bg during multiple extrusions and to confirm if cross-linking is taking place and contributing to the reduction of crystallinity in the contaminated bottle grades rPET, it is of paramount importance to understand the influence of the PP contaminant on the crystallization process of the rPET-bg matrix at constant cooling post multiple extrusions. The Avrami equation is well known for offering good explanations and describing the crystallization process through the mechanism of nucleation and growth of crystallites. This equation was used in our case to describe this process of crystallization isothermally to investigate what was really happening during the crystallization processes in both materials, non contaminated and contaminated, as a result of multiple extrusions and to validate the predominance of cross-linking or chains'-scission during multiple extrusions in both non contaminated and contaminated rPET-bg.

The crystallization process analysis, as shown in Figs. 6 and 7, showed sigmoid curves, as described by Avrami equation, clearly showed the influence of the PP contaminant and the crystallization dependency on multiple extrusions. The non contaminated rPET-bg curves showed random, narrow and fast crystallization with increased multiple extrusion cycles, indicating dependency of crystallization and multiple extrusion cycles and the ability of the material in withstanding thermo-mechanical degradation and that the crystallization process as a result of multiple extrusion cycles showed uniform and balanced nucleation and growth rate.

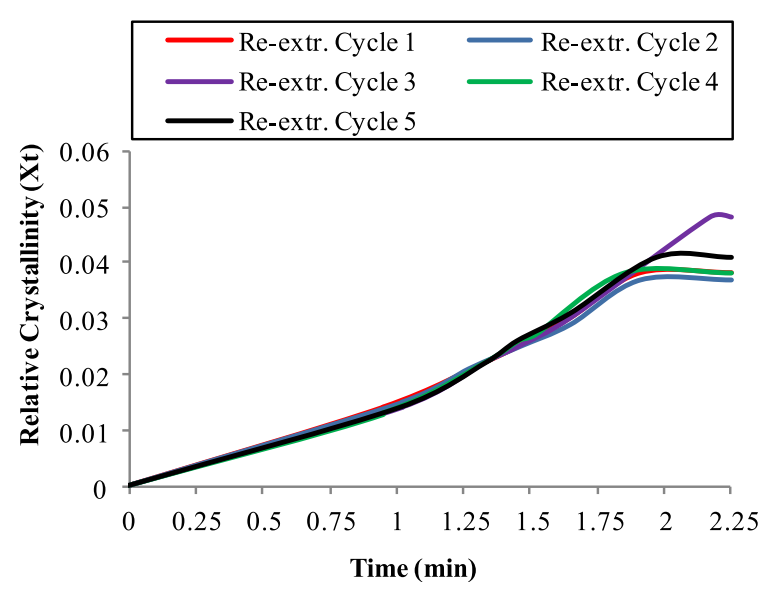

Fig. 6. rPET curves of relative cryst. Vs time various re-extrusion cycles. 


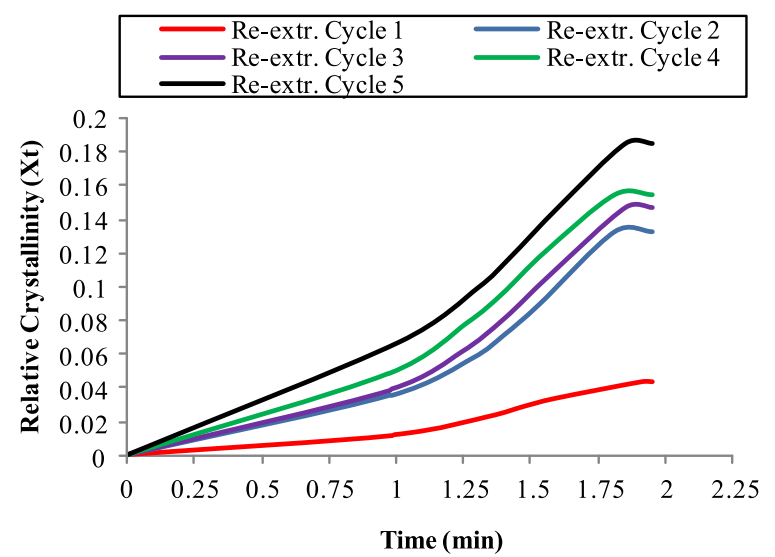

Fig. 7. $95 \% \mathrm{rPET} / 5 \% \mathrm{PP}$ curves of relative cryst. Vs time at various re-extrusion cycles.

Whereas, in the PP contaminated rPET-bg, the rate of crystallization gradually decreased uniformly with increasing multiple extrusion cycles, indicating ability of the polymer chains in maintaining disordered and entangled forms and requiring higher activation energy for disentangling and straightening.

The curves in Figs. 8 and 9 obeyed the rule of Avrami equation for the portion of the primary phase of crystallization, shown as straight lines as described by equation (2).

The values in Tables 2 and 3 and curves in Figs. 10 and 11 showed that the $\mathbf{n}$ values increased randomly with increased multiple extrusion cycles in the non contaminated rPET-bg, which indicated random sporadic nucleation due to the large allowable crystallization time. Whereas, in the PP contaminated rPET-bg, the $\mathbf{n}$ values increased at the start of the crystallization process due to the presence of small amount of short chains which crystallized faster and resulted in a primary increase, but then decreased orderly and the spherulites growth varied from disc to sporadic shapes indicating that crystallization was slower than in the non contaminated rPET-bg, indicating that by increasing multiple extrusion cycles, further cross-linking was created making it difficult for the PP contaminated rPET-bg to crystallize at lower cooling temperature even with large allowable time due cross-linking. This confirmed the exhibition of lower crystallinity in the contaminated rPET-bg due to the inclusion of the PP contaminant. Also, this indicated

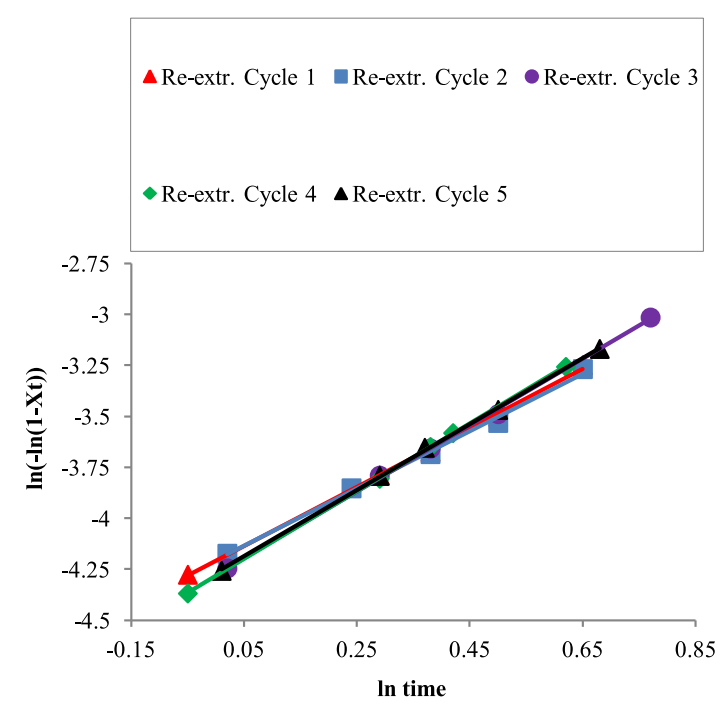

Fig. 8. Avrami double log plots of $\ln \left(-\ln \left(1-X_{t}\right)\right)$ Vs $\ln t$ of rPET at various re-extrusion cycles.

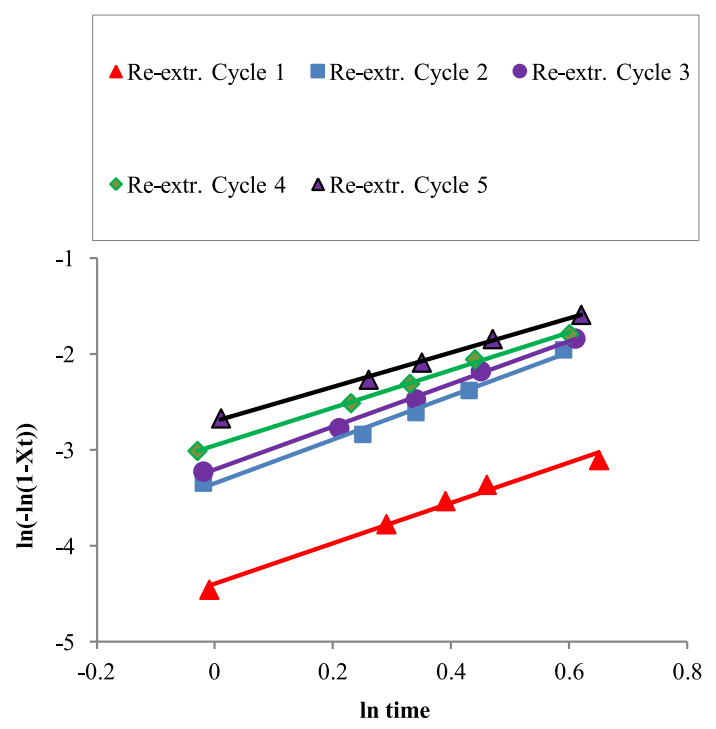

Fig. 9. Avrami double plots of $\ln \left(-\ln \left(1-X_{t}\right)\right)$ Vs $\ln t$ of $95 \%$ rPET/5\% PP at various reextrusion cycles.

Table 2

Avrami's $\mathbf{n}$ and $\mathbf{Z}_{\mathbf{t}}$ of rPET

\begin{tabular}{lllll}
\hline Extrusion cycles & $\mathrm{n}$ & Nucleation characteristics & Type & $\mathrm{Z}_{\mathrm{t}}$ \\
\hline 1 & 1.451 & Sporadic nuclei & $1 \mathrm{D}^{\mathrm{a}}$ & 0.015 \\
2 & 1.404 & Sporadic nuclei & $1 \mathrm{D}^{\mathrm{a}}$ & 0.015 \\
3 & 1.627 & Sporadic nuclei & $1 \mathrm{D}^{\mathrm{a}}$ & 0.014 \\
4 & 1.664 & Sporadic nuclei & $1 \mathrm{D}^{\mathrm{a}}$ & 0.014 \\
5 & 1.619 & Sporadic nuclei & $1 \mathrm{D}^{\mathrm{a}}$ & 0.014 \\
\hline
\end{tabular}

a Saturated site.

that the non contaminated rPET-bg was crystallizing faster. Both sporadic nucleation and fast crystallization indicated the tendency of the non contaminated and contaminated rPET-bg in remaining amorphous as shown by the very low $\mathbf{Z}_{\mathbf{t}}$ values which indicated unique characteristics of transparency and clarity. Despite the large allowable crystallization time as function of low cooling rate, both materials showed tendency in crystallizing at very slow rate which confirmed that multiple extrusions contributed in strengthening the disordered nature of entangled chains making crystallization more complex. $\mathbf{Z}_{\mathbf{t}}$ values of non contaminated rPET-bg are lower than those of the PP contaminated one because the crystallization time was largely available, this could be attributed to the degree of diffusion which is a controlling factor at low temperature, whereas the rate of nucleation dominates at high temperature according to Keith and Padden [14], this diffusion rendered the growth rate extremely slow, i.e. at low cooling rate, crystallization time was large and accompanied by a lower $\mathbf{Z}_{\mathbf{t}}$. Therefore, the higher $\mathbf{Z}_{\mathbf{t}}$ values of the PP contaminated rPET-bg accounted for slow crystallization compared with fast crystallization of the non contaminated one.

Table 3

Avrami's $\mathbf{n}$ and $\mathbf{Z}_{\mathbf{t}}$ of $95 \% \mathrm{rPET} / 5 \% \mathrm{PP}$.

\begin{tabular}{lllll}
\hline Extrusion cycles & $\mathrm{n}$ & Nucleation characteristics & Type & $\mathrm{Z}_{\mathrm{t}}$ \\
\hline 1 & 2.100 & Rod and disc growth & $1^{\mathrm{b}}$ and 2D & 0.012 \\
2 & 2.270 & Rod and disc growth & $1^{\mathrm{b}}$ and 2D & 0.034 \\
3 & 2.230 & Rod and disc growth & $1^{\mathrm{b}}$ and 2D & 0.041 \\
4 & 1.960 & Sporadic nuclei 1D & 0.052 & \\
5 & 1.790 & Sporadic nuclei 1D & 0.067 & \\
\hline
\end{tabular}

a Saturated site.

b Continuous nucleation. 


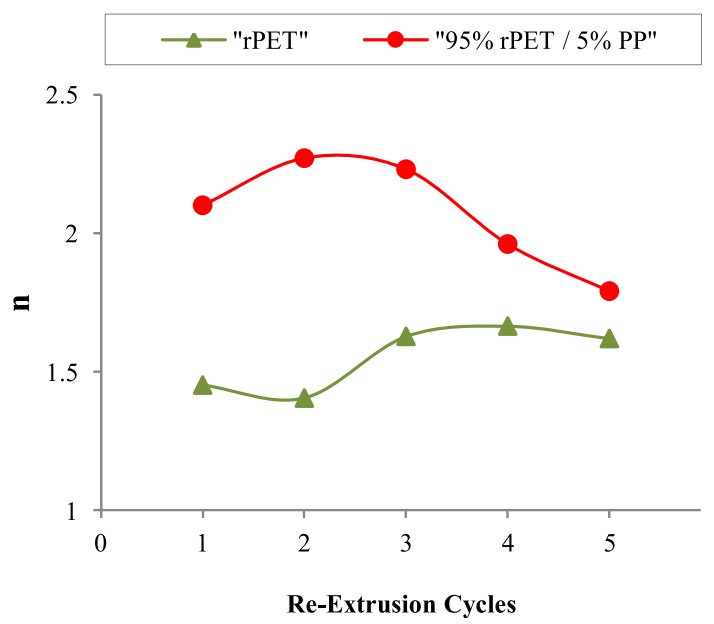

Fig. 10. Curves of Avrami's exponent, n, at various re-extrusion cycles.

As a result of this body of work it has been possible to show that the presence of 5\% PP in the rPET-bg matrix does have an impact on the crystallinity of the rPET-bg. This is confirmed by the DSC work performed on PP contaminated rPET-bg at various re-extrusions cycles (multiple extrusions) and low constant cooling rate and also the Avrami equation which confirmed the outcome of this research work. It was shown that the inclusion of small amounts of 5\% PP influenced the thermal characteristics of the rPET matrix, especially crystallinity which was disrupted and reduced as a result of the generated cross-linking. Furthermore, the Avrami equation confirmed that the crystallization process of the contaminated rPET-bg with small amount of PP depended on many influencing factors, such as impurities and the processing history but fully depended on the nucleation mechanism and the spherulite growth rates. The crystallisation process of the contaminated rPET-bg with PP indicated that nucleation and molecular mobility were competing together and that molecular mobility in the contaminated rPET-bg increased with increasing temperature, whereas nucleation rate decreased with increasing temperature. Throughout the crystallization process, the small amount of PP contaminant, played dominant role in lowering the chain mobility of the rPET-bg due to the cross-linking generated during multiple extrusions, which contributed in decreasing the nucleation speed and growth and as a result decreasing crystallinity; reducing crystallinity in PET bottle manufacturing is of paramount importance in enhancing bottles' clarity or transparency with respect to

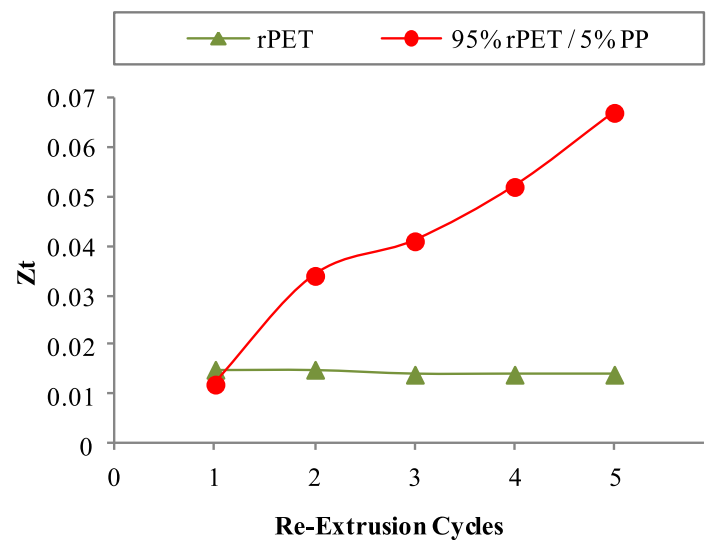

Fig. 11. Curves of Avrami's rate constant, $\mathrm{Zt}$, at various re-extrusion cycles. related properties which are required in bottle manufacturing, such as the degree of toughness and resistance to failure under stress of the produced bottles, especially during storage. A crystallinitytoughness requirement should be taken in consideration when designing bottles and products with minimum crystallinity. From industrial perspective, recyclers of post-consumer polymeric materials, such as PET or polyolefins, are continuously spending enormous manpower and financial cost in tackling the problem of contamination and its impact on the final produce, especially in the bottle manufacturing field where the primary priority is the continuous processes' optimization to reduce and minimize crystallinity. The growth of recycling post-consumer polymeric materials means that polyolefin contaminants, especially PP, must be looked at not suspiciously but with certain degree of potential reusability to enhance the recycling capabilities, reaching environmental protection targets and reducing overall financial costs. Recycling is the future of manufacturing in the field of polymers and recycling involves multiple extrusions for cost effectiveness and the confirmation of cross-linking creation as a result of multiple extrusions is of paramount importance in tackling crystallinity related complications.

\section{Conclusions}

1 The crystallinity of the PP contaminated bottle grades rPET decreased with increased extrusions cycles and the crosslinking predominated over chain scission during multiple extrusions.

2 The crystallization process of the contaminated rPET-bg with 5\% PP depended on many influencing factors, such as impurities, cross-linking and extrusion cycles but fully depended on the nucleation mechanism and the spherulite growth rates and that nucleation and molecular mobility were competing together and that molecular mobility in the polymer melt increased with increasing temperature, whereas nucleation rate decreased with increasing temperature. And during crystallization, the 5\% PP lowered the chain mobility of the rPET-bg as a result of the cross-linking generated during multiple extrusions, which contributed in decreasing the nucleation speed and growth rate and decreased crystallinity.

\section{Acknowledgements}

The authors are grateful to Nick Cliffe at Closed Loop Recycling Limited for supplying the recycled PET flakes used in this research work. Also, the members of university technical staff are gratefully acknowledged.

\section{References}

[1] Kim SH, Ahn SH, Hirai T. Crystallisation kinetics and nucleation activity of silica nanoparticle filled poly(ethylene 2,6 naphtalene). Polym 2003;44(19): 5625-34.

[2] Jabarin SA. Crystallization kinetics of polyethylene terephthalate. II. Dynamic crystallization of PET. J Appl Polym Sci 1987;34(1):97-102.

[3] Tant R, Culberson WT. Effect of molecular weight on spherulite growth rate of poly(ethylene terephthalate) via real-time small angle light scattering. Polym Eng Sci 1993;33(17):1152-6.

[4] Paci M, La Mantia FP. Competition between degradation and chain extension during processing of reclaimed poly(ethylene terephthalate). Polym Degrad Stab 1998:61(3):417-20.

[5] Torres N, Robin JJ, Boutevin B. Study of thermal and mechanical properties of virgin and recycled poly(ethylene terephthalate) before and after injection molding. Eur Polym J 2000;36(10):2075-80.

[6] Pospišil J, Horák Z, Kruliš Z, Nešpůrek S, Kuroda Si. Degradation and aging of polymer blends I. Thermomechanical and thermal degradation. Polym Degrad Stab 1999;65(3):405-14. 
[7] Camacho W, Karlsson S. Assessment of thermal and thermo-oxidative stability of multi-extruded recycled PP, HDPE and a blend thereof,Polym. Degrad Stab 2002;78(2):385-91.

[8] Badía JD, Vilaplana F, Karlsson S, Ribes-Greus A. Thermal analysis as a quality tool for assessing the influence of thermo-mechanical degradation on recycled polyethylene terephthalate. Polym Test 2009;28(2):169-75.

[9] Assadi R, Colin X, Verdu J. Irreversible structural changes during PET recycling by extrusion. Polym 2004;45(13):4403-12.

[10] Nait-Ali LK, Colin X, Bergeret A. Kinetic analysis and modelling of PET macromolecular changes during its mechanical recycling by extrusion. Polym Degrad Stab 2011;96(2):236-46.
[11] Closed Loop Recycling Ltd, http://www.closedlooprecycling.co.uk/ [accessed 30.09.09].

[12] Sperling LH. Introduction to physical polymer science. 2nd ed. Singapore: John Wiley and Sons; 1993. p. 232-5.

[13] Lauritzen Jr John I, Hoffman John D. Extension of theory of growth of chainfolded polymer crystals to large undercoolings. J Appl Phys 1973;44: 4340-53.

[14] Keith HD, Padden Jr FJ. Spherulitic crystallization from the melt. I. Fractionation and impurity segregation and their influence on crystalline morphology. J Appl Phys 1964;35:1270. 